\title{
Lexis
}

Journal in English Lexicology

13 | 2019

Lexicon, Sensations, Perceptions and Emotions

\section{Le commentaire œnologique ou comment déguster le vin par les mots}

Audrey Moutat

\section{(2) OpenEdition}

1 Journals

Édition électronique

URL : http://journals.openedition.org/lexis/3026

DOI : $10.4000 /$ lexis.3026

ISSN : 1951-6215

Éditeur

Université Jean Moulin - Lyon 3

Référence électronique

Audrey Moutat, «Le commentaire œnologique ou comment déguster le vin par les mots », Lexis [En ligne], 13 | 2019, mis en ligne le 14 mars 2019, consulté le 10 décembre 2020. URL : http:// journals.openedition.org/lexis/3026; DOI : https://doi.org/10.4000/lexis.3026

Ce document a été généré automatiquement le 10 décembre 2020.

\section{(c) (i) (9)}

Lexis is licensed under a Creative Commons Attribution-NonCommercial-NoDerivatives 4.0 International License. 


\title{
Le commentaire œnologique ou comment déguster le vin par les mots
}

\author{
Audrey Moutat
}

\section{Introduction}

1 La dégustation œnologique, qui consiste à analyser et à décrire les propriétés organoleptiques des vins, s'inscrit dans une pratique communicationnelle doublement problématique dans la mesure où elle fait appel à une terminologie qui, d'une part, ne fait pas consensus au sein de la communauté des experts et qui, d'autre part, est jugée abstraite, voire pompeuse, par les profanes. En effet, certains descripteurs utilisés en dégustation voient leur contenu sémantique varier d'une région à l'autre. Les termes employés sont souvent galvaudés, une entrée lexicale étant définie par une autre, ellemême décrite en termes d'une autre, etc. En outre, la terminologie adoptée relève de domaines sémantiques variés, si bien que les non-spécialistes la considèrent comme rhétorique, poétique et dénuée de sens. Parmi les images associées au vin, on retrouve celles du contenant («creux», "plein», «épais»), de l'architecture (à travers les descripteurs "charpenté», «solide», «construit»), ou encore celles relatives à l'être humain (envisagé dans ses aspects physiques - «décharné », " maigre », «élégant», « raffiné »- psychologiques - « agressif », « nerveux », « discret », « franc »- ou sociaux «noble », « riche », « puissant »).

2 Il est vrai que si de tels descripteurs peuvent dans un premier temps perturber le public non averti en matière de dégustation, nous montrerons que leur caractère imagé est motivé et que ces termes peuvent s'avérer déterminants dans le travail interprétatif du néophyte. En effet, les contenus associés à ces descripteurs peuvent favoriser la construction des représentations des propriétés organoleptiques du vin ainsi décrit, en raison de traits de signification partagés entre les deux domaines sémantiques convoqués. Nous verrons également que si une telle transversalité du sens s'avère possible, c'est 
parce que, sur le plan sensible, des phénomènes relevant de domaines sensoriels différents peuvent partager des traits fondamentaux et manifester une communauté d'organisation.

\section{La fonction communicationnelle des mots du vin}

3 Le pouvoir communicationnel du lexique de la dégustation a été démontré en neurosciences et en psychologie cognitive par D. Valentin, S. Chollet et H. Abdi [2003]. En soumettant deux groupes d'individus, l'un expert en dégustation, l'autre novice, à des tests de description d'une série de vins, sur la base d'un lexique identique mis à leur disposition ${ }^{1}$, puis d'appariement de ces mêmes vins à partir des textes ainsi produits, l'équipe [2003:1] a observé que si «les descriptions effectuées par les experts sont plus précises et conduisent à de meilleures performances d'appariement que celles effectuées par les novices", les résultats d'appariement de ces novices sont eux aussi meilleurs lorsqu'ils opèrent à partir des descriptions d'experts alors qu'ils ne sont pourtant pas familiarisés avec ce genre textuel. Les résultats de cette expérience permettent ainsi de tirer ce double constat : (i) premièrement, les experts sont dotés d'une meilleure capacité à décrire les traits essentiels et caractéristiques des vins; les termes sélectionnés permettent aux sujets novices de concentrer leur attention sur ces propriétés caractéristiques. En effet, les experts décomposent le nez et la bouche en leurs différents constituants aromatiques qu'ils décrivent séparément, avec des descripteurs monodimensionnels ${ }^{2}$, alors que les novices procèdent à des descriptions holistiques, en employant des termes multidimensionnels ${ }^{3}$. (ii) De ce premier constat en découle un second : novices et experts ont des représentations perceptives semblables et s'opposent alors dans l'interprétation des dimensions de leurs représentations.

Dès lors, la véritable problématique de la pratique de la dégustation se situe dans les inférences et les opérations interprétatives effectuées par le novice lors de la verbalisation de ses stimuli. D'où le caractère paradoxal du lexique de la dégustation: comment expliquer le fait que les novices comprennent la langue du vin sans la parler? Comment parviennent-ils à construire des impressions référentielles (Rastier [1987]) partielles sur le vin à partir d'un lexique qu'ils ne maitrisent pas ?

\section{Commentaires de dégustation et interprétation}

5 Afin d'évaluer la valeur communicationnelle des mots du vin, nous situerons notre analyse dans un contexte de saisie différent de celui de l'expérience pré-citée, en portant notre attention sur la lecture du commentaire de dégustation en l'absence de stimulus. Notre objectif est de comprendre comment un lecteur non spécialiste de la dégustation parvient à construire une représentation mentale globale des propriétés olfactogustatives d'un vin, à partir de ses seules facultés interprétatives.

\subsection{Présentation de l'objet d'étude}

6 Pour ce faire, nous étudierons un ensemble de commentaires de dégustation extraits de différentes revues « œnologiques » parues en kiosque entre 2003 et 2018 : Gilbert \& Gaillard ,Cuisine et Vins de France, La Revue du vin de France, Vins \& Gastronomie et Terre de Vins. 
Guide pratique pour l'achat de vins et leurs accords avec les mets, Cuisine et Vins de France consacre ses articles à la cuisine et à l'œnologie, aux domaines de production et aux techniques de vinification des vins dégustés. Accessibles, ses commentaires de dégustation adoptent un style fortement imagé et une rhétorique ${ }^{4}$ engageant une relation de proximité avec le lecteur.

Magazine de «l'art de vivre», Vins \& Gastronomie se consacre exclusivement à «l'excellence gourmande, viticole, touristique ${ }^{5}$ en défendant des valeurs de raffinement, dans le respect de la tradition, du patrimoine et du terroir. Il dispense l'actualité œnologique, gastronomique et touristique en faisant appel à des sommeliers réputés, de grands chefs ou encore de hauts lieux de l'hôtellerie de luxe. Les commentaires de dégustation sont à l'image de son éthique : dénué de toute particularité rhétorique, ils sont synthétiques et respectent le genre discursif de la fiche de dégustation.

Revue œnotouristique, Terre de vins propose de découvrir les crus et les cuvées à travers une exploration des paysages viticoles. Typicité de terroir, géothermie, méthodes de viticulture... tels sont les différents thèmes abordés. Bien qu'adressée à un public d'initiés, cette revue se laisse parfois aller à quelques enjolivements stylistiques au sein de ses commentaires de dégustation.

Spécifiquement dédiée à l'évaluation et la sélection des vins, Gilbert \& Gaillard s'adresse à un public d'amateurs confirmés. Cette revue informe son lecteur sur les nouveautés et dispense des conseils avisés sur la conservation des vins en cave. Les commentaires de dégustation sont synthétiques, articulés en trois phases (robe, nez, bouche), et sans jeux de style.

11 Ouverte à un large public, du simple amateur au fin connaisseur, La Revue du vin de France est exclusivement consacrée au vin, de la viticulture à la gestion de la cave, en passant par des préconisations pour une dégustation optimale. Guide d'achat, elle oriente les choix de ses lecteurs à travers des commentaires de dégustation concis et incisifs, à faible charge rhétorique.

\subsection{Lexique aromatique et terminologie expérientielle}

12 À la différence de D. Valentin, S. Chollet et H. Abdi [2003], nous ne porterons pas notre intérêt sur les descripteurs de la roue des arômes, autrement dit sur ceux qui décrivent les qualités sensorielles du vin en se référant à une classe ou une source aromatique ( fruité », «floral », «boisé », « fraise ", « cassis », « mûre », etc.). Nous proposons en revanche de nous concentrer sur les descripteurs dits « expérientiels » qui réfèrent à la structure du vin, dont les différentes propriétés lui confèrent une certaine " morphologie » ${ }^{6}$. Il s'agit de descripteurs tels que " fin », « délié », « dense », « dur », etc., qui soulèvent des difficultés interprétatives supérieures, comme peuvent en attester ces quelques commentaires de dégustation, extraits de La Revue du vin de France (notée RVF) :

(1) Robe soutenue, joli nez de fraises, bouche équilibrée, élégante, charnue, excellents tanins, terroir bien marqué : exemplaire pour un « villages ».

(2) La robe est profonde, brillante et nette. Au nez comme en bouche, il s'exprime dans un style flatteur, velouté, déjà ouvert et néanmoins construit pour tenir, comme le rappelle la finale charpentée, sur les arômes de jeunesse du mourvèdre (70\%). 
(3) Robe acajou, éclatante. Très belle expression aromatique, complexe et élégante, avec du cuir de luxe, du fruit rôti, des notes de tabac blond, de grillé, de jus de viande. Dense, très velouté, avec une trame tannique fondue mais encore debout. Un vin en pleine forme. On peut boire.

(4) Nez complexe, puissant et racé, avec un joli fruit, des notes épicées et minérales. Bouche dense, belle trame tannique, joli fruit et très bon potentiel.

(5) Nez riche, épanoui et complexe, avec une belle qualité de fruit et des notes épicées tendres; bouche puissante, dense, profonde, avec une très belle qualité de boisé, une trame tannique très solide et aussi une petite pointe de fermeté. Devra se fondre. vin en ses propriétés spécifiques et permet ainsi d'en déterminer la morphologie sensible. Il a également la particularité d'être fortement imagé en renvoyant à des domaines sémantiques parfois très éloignés de ceux de l'œnologie. Dans le premier commentaire, les descripteurs anthropomorphiques «équilibrée ", «élégante» et "charnue » assimilent la bouche du vin dégusté à une personne en vertu de ses propriétés physiques et psychologiques. Dans le second exemple, en revanche, c'est à une construction architecturale que les propriétés organoleptiques sont rapprochées, le descripteur « profond » pouvant renvoyer à la superficie des pièces qui la constituent, « ouvert » à ses différentes fenêtres et portes, "construit» et "charpentée » à la robustesse de l'ensemble. Ces connexions inter-isotopiques, qui attribuent à une isotopie les propriétés d'une autre, créent une démultiplication des sphères textuelles par une superposition de plusieurs images sur un même référent. Dans les commentaires 3,4 et 5, ces corrélations se complexifient dans une relation ternaire où le nez est personnifié par des descripteurs anthropomorphiques qui lui confèrent des propriétés psychologiques ("complexe», « épanoui »), physiques (« racé », « élégant»), ainsi qu'une distinction sociale (« riche », «puissant »). La bouche est quant à elle appréhendée sous l'angle d'une construction architecturale en termes de volume (« dense », « profond ») et de résistance (« solide »).

Le commentaire de dégustation se présente ainsi comme un énoncé polyisotopique animé par une tension discursive. Cette dernière tient en la coexistence de plusieurs isotopies génériques ${ }^{7}$ concurrentes associées au sein d'une même relation prédicative: dans le second commentaire, les qualités de l'argument isotopant (autrement dit la robe, le nez et la bouche) se trouvent définies à travers les propriétés d'un prédicat allotope ${ }^{8}$ relevant d'un autre domaine sémantique (la construction architecturale). Si l'impression référentielle ${ }^{9}$ est déterminée par l'isotopie œnologique, assertée par le contexte de production de l'énoncé (la dégustation), son entour textuel (la revue à l'intérieur de laquelle il est publié) et son genre (commentaire de dégustation), le caractère imagé de la seconde isotopie (construction architecturale) crée un décrochage textuel dressant une analogie sous-jacente où le vin devient profond, ouvert, construit et charpenté comme une construction architecturale. Bien que déroutante pour le lecteur non spécialisé en œnologie, la connexion de ces domaines sémantiques s'avère pourtant motivée ; elle est assurée par les sèmes spécifiques inhérents ${ }^{10}$ communs aux deux domaines sémantiques indexés et qui, en déterminant les tracés des parcours interprétatifs à suivre, garantiront l'impression référentielle œnologique : 
Tableau 1 : Sèmes spécifiques communs à $i_{1}=/ / v i n / /$ et $i_{2}=/ /$ construction architecturale//

\begin{tabular}{|c|c|c|}
\hline Sémèmes sur $\mathrm{i}_{2}$ & Sémèmes sur $i_{1}$ & Sèmes spécifiques communs \\
\hline 'profonde 1 ' & 'profonde ${ }_{2}$ ' & /intensité/ \\
\hline 'ouvert,' & 'ouvert 2 ' & /étendue/ - /non résistance/ \\
\hline 'construit' & 'construit 2 ' & /multiplicité/ - /assemblage/ \\
\hline 'charpentée 1 ' & 'charpentée ${ }_{2}$ ' & /multiplicité/ - /cohésion/ - /équilibre/ \\
\hline
\end{tabular}

\subsection{Parcours interprétatif et passerelle sémique}

15 Afin de comprendre comment opère cette démarche interprétative, nous avons mené nos analyses en nous appuyant sur la sémantique interprétative de F. Rastier [1987]. Cette méthode permet d'identifier les structures sémantiques constitutives du signifié de chaque unité lexicale et de mettre en évidence les procédures engagées dans la construction du contenu sémantique de l'ensemble du texte. Cette structuration repose sur le concept de sème, unité minimale de signification, qui permet tantôt d'indexer ces unités lexicales dans des classes sémantiques (on parle alors de "sème générique "), tantôt de les distinguer au sein d'une même classe en affichant leur singularité (telle est la fonction des « sèmes spécifiques »).

Étudions à présent ce commentaire extrait de la RVF :

(5) Nez riche, épanoui et complexe, avec une belle qualité de fruit et des notes épicées tendres; bouche puissante, dense, profonde, avec une très belle qualité de boisé, une trame tannique très solide et aussi une petite pointe de fermeté. Devra se fondre.

17 Sur le plan thématique, le sujet interprétant identifie, selon ses propres connaissances et références personnelles, les quatre isotopies ${ }^{11}$ mésogénériques ${ }^{12}$ suivantes :

18 - L'isotopie du vin ${ }^{13}$, dominante, sur laquelle se fonde l'impression référentielle du lecteur et qui indexe les sémèmes ${ }^{14}$ propres à la pratique de la dégustation et/ou aux propriétés organoleptiques du vin : 'nez' ${ }^{15}$, 'fruits', 'notes épicées', 'boisé', 'tannique' ;

- L'isotopie générique de l'être humain (personnification), saisie à travers ses propriétés psychologiques ('épanoui', 'tendre') et sociales ('riche'16, 'puissante') ;

- L'isotopie générique du volume ('dense', 'profonde');

- L'isotopie générique du bâtiment, le sémème 'solide' y étant indexé par contagion sémique perpétrée par le sémème 'trame'.

19 À cela s'ajoute le sémème de l'unité lexicale « complexe ", sémème anisotope, c'est-à-dire qui ne relève d'aucun domaine sémantique particulier et qui, de ce fait, peut être aisément transposable d'un domaine sémantique à l'autre.

20 Ces isotopies entrelacées ${ }^{17}$ constituent des structures dénominatives imagées qui, en dépit de l'incompatibilité de leurs sèmes isotopants, poussent le sujet interprétant à dresser des analogies entre les propriétés de l'argument et celles du prédicat :

«Le vin est riche, épanoui, tendre et puissant comme peut l'être une personne.»

«Le vin est dense et profond comme un volume.»

« Le vin est solide comme une structure architecturale.» 
Tableau 2 : Connexions inter-isotopiques $:_{1} \mathrm{i}_{1}=/ / \mathrm{vin} / /$ et $\mathrm{i}_{2}=/ /$ être humain// référentielles organoleptiques. Il se déroule en trois temps : prédicative. d'extraire les traits spécifiques de leur sémantème ${ }^{18}$. l'isotopie dominante.

D’où les grilles sémiques suivantes :

Le travail interprétatif consiste ainsi à déterminer le fondement de ces connexions interisotopiques dans le but de rétablir la cohérence textuelle et de construire des impressions

1. Lors de la première phase de lecture, le sujet interprétant identifie la tension discursive instaurée par la coexistence de ces isotopies concurrentes au sein d'une même relation

2. Puis il se tourne vers le contenu des sémèmes indexés sur l'isotopie comparante afin

3. Enfin, il procède à un transfert de ce sémantème sur les sémèmes de l'isotopie comparée qu'il interprète au regard de leur classème ${ }^{19}$ afin de reconstruire l'intégralité de

$$
\text { Tableau } 2 \text { : Connexions inter-isotopiques }{ }_{1}: i_{1}=/ / \mathrm{vin} / / \mathrm{et}_{2}=/ / \text { être humain// }
$$

\begin{tabular}{|c|c|c|}
\hline Sémèmes sur $\mathrm{i}_{2}$ & Sémèmes sur $\mathrm{i}_{1}$ & Sèmes spécifiques communs \\
\hline 'riche ${ }_{1}$ ' & 'riche ${ }_{2}$ ' & /multiplicité/, /mélioratif/, /intensité/ \\
\hline 'épanoui 1 ' & 'épanouiz' & /étendue/,/intensité/, /tonicité/, /mélioratif/ \\
\hline 'tendre 1 ' & 'tendre' & /atonie/,/non résistance/ \\
\hline 'puissant 1 ' & 'puissant ${ }_{2}$ ' & /tonicité/, /force/, /multiplicité \\
\hline
\end{tabular}

Tableau 3 : Connexions inter-isotopiques ${ }_{2}: i_{1}=/ / v i n / /$ et $i_{3}=/ /$ volume $/ /$

\begin{tabular}{|c|c|c|}
\hline Sémèmes sur i 3 & Sémèmes sur $\mathrm{i}_{1}$ & Sèmes spécifiques communs \\
\hline 'dense ${ }_{1}^{\prime}$ & 'dense ${ }_{2}^{\prime}$ & /intensité/, /multiplicité/, /liaison/, /saturation/ \\
\hline 'profond ${ }_{1}$ ' & 'profond 2 ' & /étendue/,/multiplicité/,/distance/ \\
\hline
\end{tabular}

Tableau 4 : Connexions inter-isotopiques ${ }_{3}: \mathrm{i}_{1}=/ / \mathrm{vin} / /$ et $\mathrm{i}_{4}=/ /$ construction $/ /$

\begin{tabular}{|c|c|c|}
\hline Sémèmes sur $\mathrm{i}_{4}$ & Sémèmes sur $\mathrm{i}_{1}$ & \multicolumn{1}{c|}{ Sèmes spécifiques communs } \\
\hline 'solide $_{1}{ }^{\prime}$ & 'solide & ${ }_{2}$ \\
\hline
\end{tabular}

Le sémème 'profond', par exemple, actualise le sème mésogénérique /volume/, univers de référence à partir duquel va opérer le travail interprétatif du lecteur qui va isoler les sèmes spécifiques du sémème comparant (/étendue/ et /distance/), avant de les transférer sur le sémème comparé. Ce transfert de sens met ainsi en évidence l'exercice d'une molécule sémique ${ }^{20}$ en flottaison, qu'il semble impossible de récrire en un sémème propre à la pratique de la dégustation. Cette molécule, constituée des sèmes spécifiques inhérents au sémème de l'isotopie-source, est strictement identique à celle du descripteur ${ }^{21}$ de l'isotopie-cible. Ainsi, en l'absence de terme propre à la pratique de la dégustation, les sujets interprétants sont contraints d'accepter l'existence d'un sémème 'profond '. Ce nouveau sémème intègre le noyau sémique extrait à partir de l'isotopie-source, et s'enrichit ensuite, sur l'isotopie-cible, des traits afférents contextuels ${ }^{22}$ liés à la pratique de la dégustation. 




Isotopie 2 = 'profond 1' (volume)

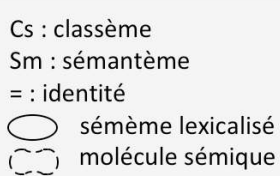

$\mathrm{Sm} \cdot \mathrm{sem}$

sémème lexicalisé

molécule sémique

molécule sémique en flottaison nous permet ainsi d'observer que le lexique de la dégustation s'est construit au moyen d'une "passerelle sémique » (A. Moutat [2015]). Cette dernière assure le passage d'un dialecte (autrement dit le langage ordinaire / sens commun), à un sociolecte - langage de la pratique de la dégustation - par la médiation d'une molécule de sèmes spécifiques qui, extraite du sémème d'usage courant, partage des propriétés sémantiques avec le sémème d'usage spécifique. Grâce à cette molécule de sèmes nucléaires communs, la passerelle sémique assure un décrochage sémique par rapport à un univers de référence usuel pour ouvrir et institutionnaliser un nouveau champ de connaissances qui lui est néanmoins connecté.

Figure 2 : Passerelle sémique

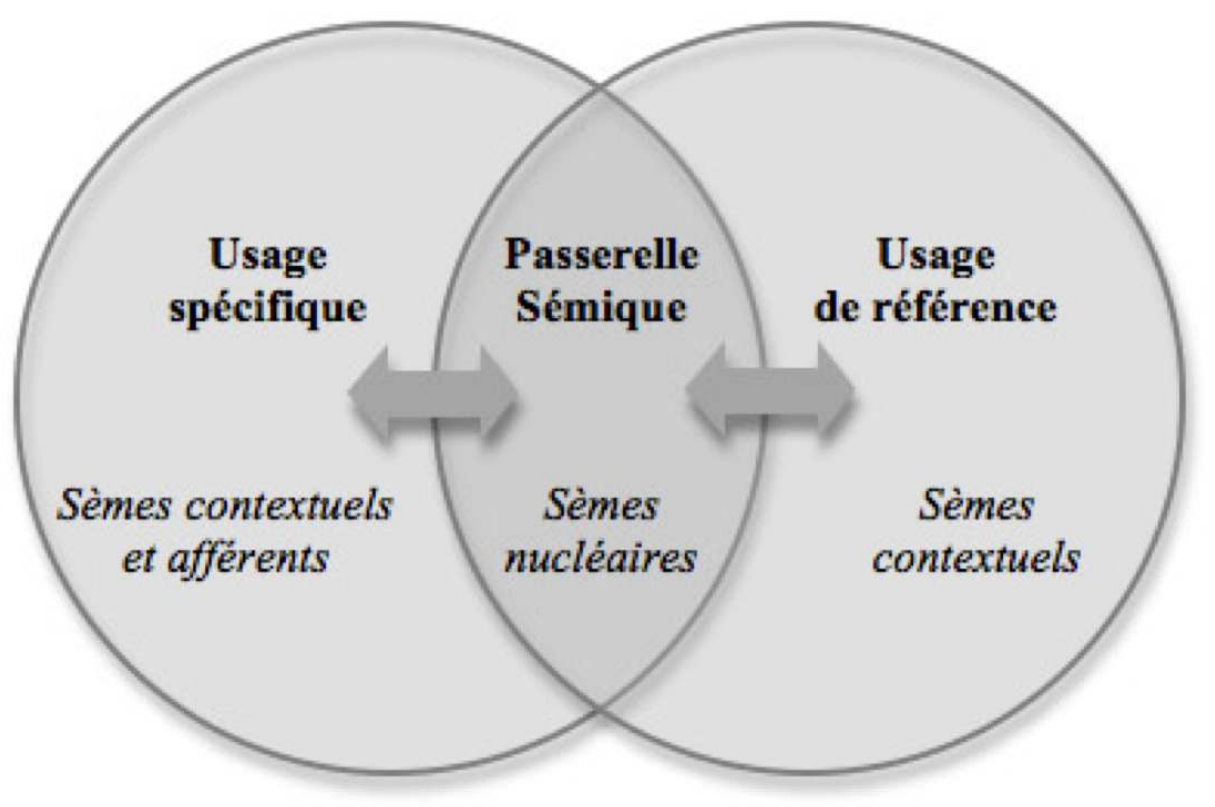

Cette procédure de génération d'une terminologie telle que celle de la dégustation des vins correspond ainsi à une étape d'un processus d'abstraction. La molécule sémique 
extraite du lexique usuel s'autonomise pour se fixer sur un second domaine sémantique qu'elle formalise. Elle fonctionne, en ce sens, comme un «opérateur de montée en généricité ", pour reprendre l'expression de Y.-M. Visetti ${ }^{23}$, opérateur censé révéler un canevas phénoménal qui sous-tend la connaissance du monde sensible et permet de ce fait de l'élever en champ de connaissance ${ }^{24}$.

Dès lors, si les propriétés organoleptiques d'un vin peuvent être décrites dans les termes de propriétés sensibles relevant d'un autre ordre sensoriel, c'est parce que ces phénomènes sensibles partagent des propriétés expérientielles communes.

\section{La dégustation, jeu de transferts expérientiels}

Tâchons donc de déterminer quelles sont ces "propriétés expérientielles communes ". Nous nous appuierons sur le concept d'iconicité proposé par J.-F. Bordron [2000, 2002, 2004] dans sa sémiotique de la perception.

Inscrit dans une perspective phénoménologique, ce concept part du postulat selon lequel nous ne percevons pas des objets mais des esquisses de ces objets. Ainsi, le dégustateur ne perçoit pas l'ontologie matérielle du vin (ce qui relève de la chimie), mais sa phénoménalité. Pour ce faire, il ajuste ses actes perceptifs en fonction des différents aspects sensibles du vin qui apparaissent avec plus ou moins de résistance ou de saillance. Cela se traduit par des mouvements de rotation et d'inclinaison du verre (ce qui lui permettra de déterminer la consistance des «larmes» du vin ainsi que son intensité lumineuse), des actes d'olfaction répétés, nez au-dessus du verre (afin de mesurer l'évolution des arômes et leur harmonie), ou encore la « mâche » du vin en bouche (grâce à laquelle il appréciera la consistance de sa matière ainsi que sa texture). À cet égard, J.F. Bordron reconnait le caractère dynamique des objets de sensation qui se manifestent dans le champ perceptif à travers un processus de constitution articulé en deux moments : (i) l'appréhension, où une présence sensible apparaît de manière indicielle sans que l'on puisse clairement l'identifier. En dégustation, on parlera par exemple d'un nez «fruité », ce qualificatif renvoyant à une catégorie aromatique générique qui ne permet pas encore de déterminer avec précision les éléments perçus ; et (ii) l'iconisation ${ }^{25}$ , moment où cette présence prend forme et se configure en une structure phénoménale complexe appelée "icône ». Dans notre exemple, le nez du vin se développera et apparaitra alors « sur des fruits rouges, avec des arômes de myrtille et de mûre en tête et une note de fraise des bois en retrait ».

L'icône correspond à une structure sensible articulée par les trois catégories kantiennes de la quantité (laquelle renvoie à la matière de la sensation), la qualité (correspondant à son intensité), et la relation (forme générale du phénomène). Selon J.-F. Bordron [2000:16] ces catégories « se déploient au lieu même du phénomène non pas comme les moments d'une analyse [...] mais bien comme le déploiement d'un germe en quoi consiste d'abord l'événement perceptif ». Ce germe représente la structure de base d'un phénomène sensible sous la forme d'une "molécule phénoménale ", à la manière de la représentation de Cram pour les molécules chimiques. Cet assemblage d'éléments atomiques (ici des catégories élémentaires) unis par des liaisons est ensuite susceptible de se déployer en d'autres ramifications selon la complexité de la structure sensible. Dès lors, chaque agrégat catégoriel permet de représenter les propriétés caractéristiques de chaque expérience sensible, de les comparer et de les classer. 


\section{Quantité Relation}

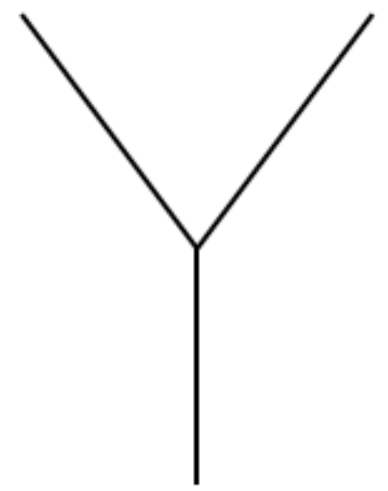

\section{Qualité}

Partant de ces considérations, revenons à présent sur le commentaire de dégustation dont nous avons analysé le parcours interprétatif, afin de dégager la structure phénoménale (ou icône) exprimée par chaque terme engagé dans la description des propriétés olfactives de ce vin.

(5) Nez riche, épanoui et complexe, avec une belle qualité de fruit et des notes épicées tendres; bouche puissante, dense, profonde, avec une très belle qualité de boisé, une trame tannique très solide et aussi une petite pointe de fermeté. Devra se fondre.

32 À cet égard, nous avons vu que ces propriétés olfactives sont décrites à l'aide de descripteurs relevant de l'isotopie de l'être humain : 'riche', 'épanoui', 'tendre', auxquels nous pouvons ajouter le sémème anisotope 'complexe' qui s'inscrit dans cette isotopie par contagion sémique.

Ainsi, le sémème 'riche' détermine l'abondance des éléments de valeur que possède un être humain ou, comme il en est question ici, le nez du vin. De ce fait, il indexe les sèmes / multiplicité/ et /intensité/ qui réfèrent respectivement, sur le plan sensible, aux catégories de la Quantité et de la Qualité. 


\section{Quantité}

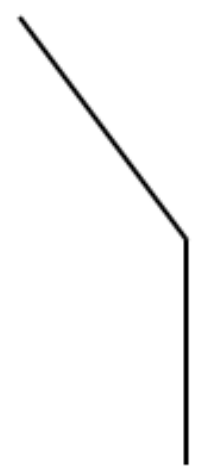

\section{Qualité}

En actualisant les sèmes spécifiques /étendue/, /intensité/ et /tonicité/, le sémème 'épanoui' renvoie à la force d'exhalaison des arômes de ce nez qui envahissent le champ perceptif. Cette structure phénoménale est assimilable au relâchement de la tension d'une personne qui gagne en sérénité. Dès lors, les sèmes /intensité/ et /tonicité/ font écho, sur le plan sémantique, à la catégorie de la Qualité, et plus particulièrement à la sous-catégorie de la Force, tandis que le sémème /étendue/ renvoie à celle de la Relation, et plus particulièrement la Direction à travers laquelle s'exprime un tel phénomène olfactif. 
Figure 5 : Structure iconique du descripteur « Épanoui »

\section{Direction}



Relation

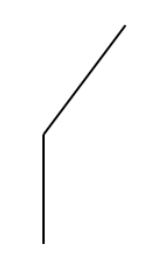

Qualité

Force

35 Quant à 'complexe', il désigne un ensemble composé d'éléments divers liés par des rapports si ténus et subtils qu'ils sont difficiles à saisir. Lorsque cet adjectif caractérise une personne, c'est pour soulever sa personnalité à multiples facettes qu'il n'est pas aisé de cerner. Ainsi, ces deux contenus mettent en exergue les sèmes spécifiques / multiplicité/, /diversité/, /connexion/ et /résistance/ qui, conjointement envisagés, renvoient à l'intégralité de la catégorie de la Quantité, à travers ses sous-catégories de la Force, la Méréologie et Répartition. 
Figure 6 : Structure iconique du descripteur « Complexe»

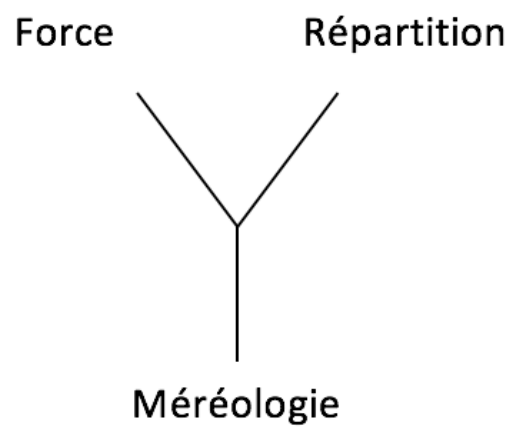

\section{Quantité}

Enfin, le sémème 'tendre' désigne une personne affectueuse qui se laisse facilement émouvoir. En dégustation, il caractérise un vin moelleux et frais, dont les tanins sont peu sensibles. Ces deux contenus manifestent le sémème de l'/atonie/ et nie toute / résistance/. Ils font ainsi référence à la catégorie de la Qualité, et notamment à sa souscatégorie : la Force. 


\section{Résistance \\ 千 \\ Force}

\section{Qualité}

37 Ainsi, la structure phénoménale complète de ce nez résulte de l'agrégation des noyaux schématiques élémentaires exprimés par chaque sémème et qui ont la particularité de mettre en évidence les propriétés phénoménales communes entre ces types d'objets sensibles : de ce fait, si ressentir une impression piquante au nez (telle que celle laissée par le vinaigre) est assimilable à l'expérience tactile d'une piqûre, c'est parce que ces deux événements sensibles partagent un noyau schématique commun qui se spécifie ensuite selon leur contexte de manifestation respectif. 
Figure 8 : Structure iconique du nez décrit dans le commentaire
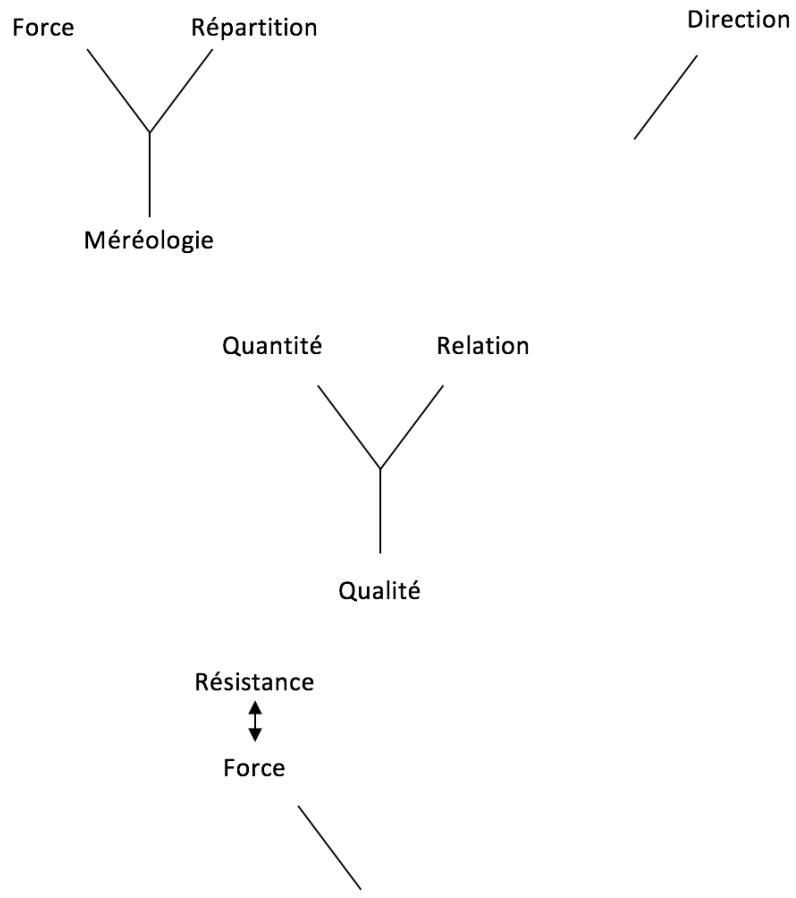

Dès lors, si le lexique de la dégustation des vins présente un caractère imagé, cela ne relève pas tant d'une visée poétique que d'une réelle correspondance phénoménale entre les propriétés du vin et ce à quoi elles sont comparées, en vertu de leurs configurations iconiques partagées. D'ailleurs, la charpente de la bouche s'avère être, à cet égard, un brillant exemple.

\section{Conclusion}

Cette brève étude aura permis de montrer la portée stratégique du lexique de la dégustation qui, en introduisant une rupture isotopique au sein du commentaire, permet de connecter deux domaines sémantiques distincts mais néanmoins dotés de sèmes spécifiques communs. Cette transversalité du sens traduit sémantiquement l'existence d'une communauté d'organisation, au niveau phénoménal, entre expériences relevant d'ordres sensoriels différents. Ces correspondances iconiques entre objets sensibles sont donc les éléments moteurs de la verbalisation de nos sens car, en se fondant sur des données expérientielles communes et plus aisément saisissables de tous, elles permettent d'accroître le potentiel communicationnel du dégustateur. Nous sommes alors conduite à inférer qu'il existerait un continuum entre perception et langage en vertu duquel des objets du monde peuvent être décrits en termes d'autres objets, et cela en raison de leurs propriétés partagées.

Le monde sensible ne serait donc pas aussi vaste qu'il n'y paraît. Il se réduirait en effet à des micro-structures expérientielles élémentaires, indépendantes de tout ordre sensoriel spécifique, et constitutives d'une strate primitive de signification commune, située à un niveau épi-sémiotique. 


\section{BIBLIOGRAPHIE}

BORDRON Jean-François, 2000, « Catégories, icônes et types phénoménologiques », La catégorisation perceptive, les frontières du Soi et de l'Autre, Visio, t. 5, 1, 7-18.

BORDRON Jean-François, 2002, « Perception et énonciation dans l'expérience gustative. L'exemple de la dégustation d'un vin », in HÉNAUlt Anne (ed.), Questions de sémiotique, Paris : PUF, coll.

«Premier cycle », 639-665.

BORDRON Jean-François, 2004, «L'iconicité ", in HÉNAULT Anne \& BEYAERT-GESLIN Anne (eds.), Ateliers de sémiotique visuelle, Paris : PUF, 121-154.

CADIOT Pierre \& VISETTI Yves-Marie, 2001, Pour une théorie des formes sémantiques. Motifs, Profils, Thèmes, Paris : PUF.

MoutAT Audrey, 2010, « Pertinence de la stylistique dans les commentaires de dégustation œnologiques ", in BOUGAULT Laurence \& WULF Judith (eds.), Stylistiques ?, Rennes : PUR, 189-206, également disponible à https://books.openedition.org/pur/40074?lang=fr

MOUTAT Audrey, 2012, «Stratégies rhétoriques et efficience communicationnelle dans les commentaires de dégustation des vins », in MISSIRE Régis (ed.), Texto!, Volume XVI, $\mathrm{n}^{\circ} 4$, disponible à http://www.revue-texto.net/docannexe/file/2908/article_audrey_moutat.pdf MOUTAT Audrey, 2015, Du sensible à l'intelligible. Pour une sémiotique de la perception, Limoges : Lambert-Lucas.

RASTIER François, 1987, Sémantique interprétative, Paris : PUF.

RASTIER François, 1989, Sens et Textualité, Paris : Hachette Supérieur.

VALENTIN Dominique, CHOLLET Sylvie \& ABDi Hervé, 2003, « Les mots du vin : experts et novices diffèrent-ils quand ils décrivent des vins ? », Corpus, Numéro 2, 183-200.

VISETTI Yves-Marie, 2013, « Le métalangage comme opérateur de phénomènes : questions pour une phénoménologie sémiotique ", Séminaire de Sémiotique des universités Paris IV Sorbonne, Limoges et Paris 8.

\section{Sitographie}

Cuisine et Vins de France : https://www.marieclaire.fr/cuisine/

Gilbert \& Gaillard : https://fr.gilbertgaillard.com

La Revue du vin de France : https://www.larvf.com

Terre de Vins : http://www.terredevins.com

Vins \& Gastronomie : https://www.vinsetgastronomie.com 


\section{NOTES}

1. En l'occurrence, il s'agissait du lexique aromatique de la roue des arômes proposée par Ann Noble, renseignée dans l'article de D. Valentin, S. Chollet \& H. Abdi [2003] comme suit: Wine Aroma Wheel Copyright (C1990, A.C. Noble.

2. Termes exprimant une note précise, spécifique, résultant d'une appréhension analytique de l'odeur : artichaut, violette, figue, réglisse...

3. Termes qui expriment une impression générale fondée sur une appréhension holistique de l'odeur : végétal, floral, fruité, épicé...

4. À propos de la stylistique et de la rhétorique dans les commentaires de dégustation œnologique, voir A. Moutat [2010] et [2012].

5. Voir à ce propos la présentation du groupe V\&G Magazines sur le site de la revue : https:// www.vinsetgastronomie.com

6. Nous considérerons ce terme dans son acception sémiotique telle qu'elle a été proposée par J.F. Bordron [2004]. Selon cet auteur, une morphologie sensible est ce qui est donné à l'expérience perceptive sous la forme d'une configuration stabilisée de catégories articulées les unes aux autres, autrement dit une structure qui se maintient dans le temps et qui présente différents traits distinctifs. Nous reviendrons sur ce point dans notre dernière partie.

7. La terminologie sémantique empruntée ici s'appuie sur la Sémantique interprétative [1987] développée par F. Rastier.

8. Deux sémèmes sont dits allotopes lorsqu'ils entretiennent une relation de disjonction en raison de l'incompatibilité de leurs sèmes respectifs.

9. Une impression référentielle est une «représentation mentale, à caractère perceptif, construite au terme du parcours interprétatif d'une séquence linguistique » (A. Moutat [2015 : 247]).

10. Selon F. Rastier [1989: 280], il s'agit d'un « sème que l'occurrence hérite du type, par défaut : par ex. /noir/ pour 'corbeau' ».

11. Selon A.-J. Greimas [1966:30], il s'agit d'un «[e]nsemble redondant de catégories sémantiques qui rend possible la lecture uniforme du récit ».

12. Il s'agit d'un sème relatif à un domaine sémantique.

13. Compte-tenu du contexte de lecture (magazine œnologique) et du genre textuel, le lecteur, même profane, reconnaît immédiatement qu'il s'agit d'un commentaire de dégustation de vin.

14. Nous convoquons une nouvelle fois la terminologie de F. Rastier [1987] pour qui le sémème est le «contenu d'un morphème » et dont la notation l'identifie par l'utilisation de guillemets fermants.

15. On notera toutefois que l'indexation de ce sémème sur l'isotopie du vin ne s'effectue pas directement et nécessite un minimum de connaissances de la part du sujet interprétant. Il en est de même pour les sémèmes 'bouche' et 'robe'. Cependant, ce point peut être tout à fait relativisé dans la mesure où, de nos jours, de tels sémèmes sont ancrés dans notre culture générale française et ne semblent pas poser de problèmes interprétatifs particuliers.

16. 'Riche' est le descripteur qui permet l'identification de l'isotopie de l'être humain. Pour le sujet interprétant concerné, il permet l'indexation, sur cette même isotopie, des adjectifs 'épanoui', 'tendre' et 'puissante' par contagion sémique.

17. Selon la terminologie de F. Rastier [1987: 276], il s'agit «d'isotopies lexicalisées dont les sémèmes alternent dans des séquences inférieures à la dimension de l'énoncé ».

18. Il s'agit de l'ensemble des sèmes spécifiques d'un sémème (noté $\mathrm{Sm}$.).

19. Le classème est l'ensemble des sèmes génériques d'un sémème (noté Cs.). 
20. «Groupe stable de sèmes, non nécessairement lexicalisé, ou dont la lexicalisation peut varier » (F. Rastier [1987: 277]).

21. Ce descripteur est en quelque sorte « virtuel » dans la mesure où il ne diffère pas du premier.

22. Par sèmes afférents contextuels, nous entendons les sèmes actualisés dans l'occurrence sémémique par une instruction contextuelle véhiculée par une détermination ou une prédication.

23. Communication prononcée par Yves-Marie Visetti lors du Séminaire de Sémiotique des universités Paris IV Sorbonne, Limoges et Paris 8, le 9 janvier 2013 : « Le métalangage comme opérateur de phénomènes : questions pour une phénoménologie sémiotique ».

24. Ceci est à rapprocher de la notion de motif développée dans P. Cadiot \& Y.-M. Visetti [2001].

25. Voir à ce propos (J.-F. Bordron [2004]).

26. Ce schéma se lit du centre vers la périphérie (à la manière de l'exfoliation sensible qu'il représente). Chaque trait continu figure une liaison entre les catégories actualisées. Chacune de ces catégories peut encore se subdiviser en sous-catégories, comme dans la figure 6 par exemple, où la catégorie de la Quantité se trouve exprimée à travers les trois sous-catégories de la Force, la Répartition et de la Méréologie, liées par une relation de dépendance interne illustrée par la conjonction de trois traits continus. Lorsqu'une catégorie ne se manifeste pas dans le phénomène sensible décrit (figure 4), son absence est matérialisée par l'absence de trait continu au sein de la représentation schématique.

\section{RÉSUMÉS}

Cette étude s'inscrit au sein d'une réflexion plus générale que nous menons depuis quelques années sur la mise en discours des perceptions. Elle cherche à remplir un double objectif : (i) déterminer comment les sujets parviennent à faire usage de la langue française dans l'expression de leurs perceptions d'une part, et (ii) comprendre comment les phénomènes sensibles ainsi décrits s'organisent en morphologies qui les configurent en structures signifiantes, d'autre part. Pour mener à bien cette entreprise, nous nous sommes tournée vers la pratique de la dégustation œnologique dont le principe consiste à interpréter, décrire et évaluer les perceptions des propriétés organoleptiques d'un vin. Nous avons ainsi collecté des commentaires de dégustation publiés dans cinq revues «œnologiques » françaises vendues en kiosque dans le but d'identifier les dispositifs communicationnels engagés par le dégustateur professionnel dans l'expression de la complexité des vins dégustés. Nous nous sommes plus particulièrement concentrée sur le lexique de la dégustation dont nous avons mesuré la pertinence grâce à une étude des parcours interprétatifs qu'ils suscitent. L'usage conjoint de la sémantique interprétative de François Rastier et de la théorie de l'iconicité de Jean-François Bordron nous a ainsi permis de soulever l'existence d'une communauté d'organisation entre la perception (qualités organoleptiques du vin) et le langage (propriétés sémantiques de la terminologie œnologique). En effet, nous constatons que ce lexique, parfois considéré comme fortement imagé, traduit sémantiquement des correspondances entre expériences perceptives relevant d'ordres sensoriels distincts.

This paper is part of a wider study on the discourse on perception I have conducted in the past few years. I had two goals: (i) on the one hand, I tried to determine how people use the French language in order to talk about their perceptions; (ii) on the other hand, I wanted to understand 
how the perceived phenomena thus described are organized into morphological forms and become significant.

To carry out this study, I decided to work on wine tasting to identify, describe and evaluate how the organoleptic qualities of a wine are perceived. I built a corpus comprising tasting notes published in five French "oenological" magazines sold in newsstands. I aimed to identify communication devices used by professional tasters to describe the complexity of the wines tasted. I particularly focused on the lexicon of taste and thus evaluated the relevance of this terminology by studying the interpretive pathways it induces. Both the interpretive semantics developed by François Rastier and Jean-François Bordron's theory of iconicity allowed me to find the common organization between perception (the organoleptic qualities of a wine) and language (the semantic properties of the wine terminology). Indeed, I noticed that this wine terminology, sometimes considered "metaphorical", is similar to the terminology relating to perceptions deriving from different senses.

\section{INDEX}

Mots-clés : sémantique interprétative, dégustation œnologique, communication sensorielle, lexique de dégustation, sémiotique, iconicité

Keywords : interpretive semantics, wine tasting, sensory communication, wine lexicon, semiotics, iconicity

\section{AUTEUR}

\section{AUDREY MOUTAT}

CeReS, Université de Limoges

audrey.moutat@yahoo.fr 